\title{
Multi-epoch $L$-band Spectroscopy of the Be Star $\mu$ Centauri Prior to Outburst
}

\author{
G. Aguayo ${ }^{1}\left(\mathbb{1}\right.$, R. E. Mennickent ${ }^{1}$, A. Granada $^{2,4,5}$, and S. Otero ${ }^{3}$ \\ ${ }^{1}$ Universidad de Concepción Departamento de Astronomía Casilla 160-C, Concepción, Chile; gusaguayo@astro-udec.cl \\ ${ }^{2}$ Department of Physics and Astronomy, The University of Western Ontario, Canada \\ ${ }^{3}$ Buenos Aires, Argentina American Association of Variable Star Observers (AAVSO) Cambridge, MA, USA \\ Received 2017 November 16; revised 2018 July 21; accepted 2018 July 23; published 2018 October 1
}

\begin{abstract}
With the aim of contributing to the understanding of the disk formation process in Be stars, we pursued a one-year spectroscopic observing campaign of the Be star $\mu$ Centauri in the $L$-band, using VLT/ISAAC. We present the nine near-IR spectra we obtained in an epoch of relative photometric quiescence prior to an outburst of $\Delta V=0.4$ magnitude. Visual estimates during the epoch of our $L$-band spectroscopy are also presented for the first time, together with the unpublished complete visual light curve between the years 1998 and 2014. We observe significant and monotonic changes in emission line strength of Bracket- $\alpha$ and Pfund- $\gamma$ lines relative to Humphreys lines, and also in the continuum slope. We interpret these observed changes in terms of important changes to the column density of the line emitting regions, moving from an optically thin to an optically thick stage just prior to a major outburst. For each observing date, we provide estimates for the column density and relative extension of the line emitting region. If the changes observed toward the end of our observing campaign were related to mass-loss changes from the central star, they would correspond to an increase in a factor of two in the mass of the disk in the innermost region. If related to the visual outburst observed one month later, the variability observed in our spectra would be the first detection of the early disk formation process in the $L$-band.
\end{abstract}

Key words: circumstellar matter - infrared: stars - stars: emission-line, Be - stars: mass-loss

Supporting material: machine-readable table

\section{Introduction}

Be stars are well known for being fast rotators, in many cases reaching velocities close to the break-up velocity (e.g., Townsend et al. 2004; Frémat et al. 2005; Martayan et al. 2007). Be stars are broadly known as B-type non-supergiant stars that show, or have shown during their lifetimes, emission in the $\mathrm{H} \alpha$ line (Jaschek et al. 1981). This emission is formed by hydrogen ionization and posterior electron recombination in a circumstellar gaseous envelope $\left(n_{e} \sim 10^{12} \mathrm{~cm}^{-3}\right)$ that has been ejected from the star by an as yet unknown mechanism. Most Be stars have variable brightness over a wide variety of timescales from hours to years, which reflects different physical processes occurring in the star and its surrounding environment. In particular, mass ejection from the central star is responsible for long-term variability, and the transient nature of the Be phenomenon is given by the formation and possible dissipation of the disk.

The origin of the mechanisms involved in the appearance of the Be phenomenon has challenged astrophysicists for 150 years, since the first observation of $\gamma$ Cas by Jesuit father Secchi in 1867. In past decades, the Be phenomenon has been reviewed in two major pieces of work, by Porter \& Rivinius (2003) and more recently by Rivinius et al. (2013).

Polarimetric data and optical interferometric observations support the hypothesis of a geometrically flattened circumstellar disk with $H / R \sim 0.1$ and $\rho / \rho_{0} \sim r^{-n}$ with $n \approx 3.5$, where $\rho_{0}$ is the disk central density and, $H$ and $R$ the disk height and radius, respectively (Rivinius et al. 2013 and references therein). An important observational insight into the behavior of mass ejection and envelope formation/dissipation comes from the identification of loops in the color-magnitude diagram

\footnotetext{
${ }^{4}$ Now at Instituto de Astrofsica de La Plata CCT La Plata, CONICET-UNLP, Paseo del Bosque S/N B1900FWA La Plata, Argentina.

${ }^{5}$ Creator of AASTeX v6.1.
}

of some outbursting Be stars (de Wit et al. 2006). These loops were modeled with an optically thick disk ejected by the star that posteriorly dissipates as an optically thin ring. This feature in the color-magnitude diagram, already noted by Dougherty et al. (1994) has been explored more recently by Haubois et al. (2012). These authors interpret this loop in terms of the different timescales of the build-up and dissipation of the disk, that is, the timescale for disk formation is shorter than the dissipation timescale. Similar conclusions were obtained by Vieira et al. (2017). A correlation between $\mathrm{H} \alpha$ emission and visual magnitude strength has been proposed by Harmanec (1983) and more recently supported by Sigut \& Patel (2013), who interpreted the correlation in terms of disks that grow in size and/or density with time. The Be star outbursts are presently being modeled within the theory of viscous decretion disks controlled by variable mass loss $\dot{M}$ and parameterized through the viscosity parameter $\alpha$ (Haubois et al. 2012; Rímulo et al. 2015).

Lee et al. (1991) constructed a viscous, geometrically thin Keplerian disk to describe the circumstellar environment of Be stars. These disks were assumed to be similar to accretion disks but with the angular momentum being supplied at the inner boundary by an uncertain mechanism, and carried away with the matter drifting outwards through the action of viscosity. This scenario became successful in describing the observed characteristics in Be stars mentioned above and has been developed since then by Porter (1999), Bjorkman (1997), Okazaki et al. (2002), Bjorkman \& Carciofi (2005), Carciofi et al. (2012), Haubois et al. (2014), Draper et al. (2014), and Vieira et al. (2017) among others.

The results obtained with the viscous decretion disk model suggest that the process of mass ejection and subsequent envelope development can affect the behavior of emission line decrements, in particular in the infrared spectral region. While 


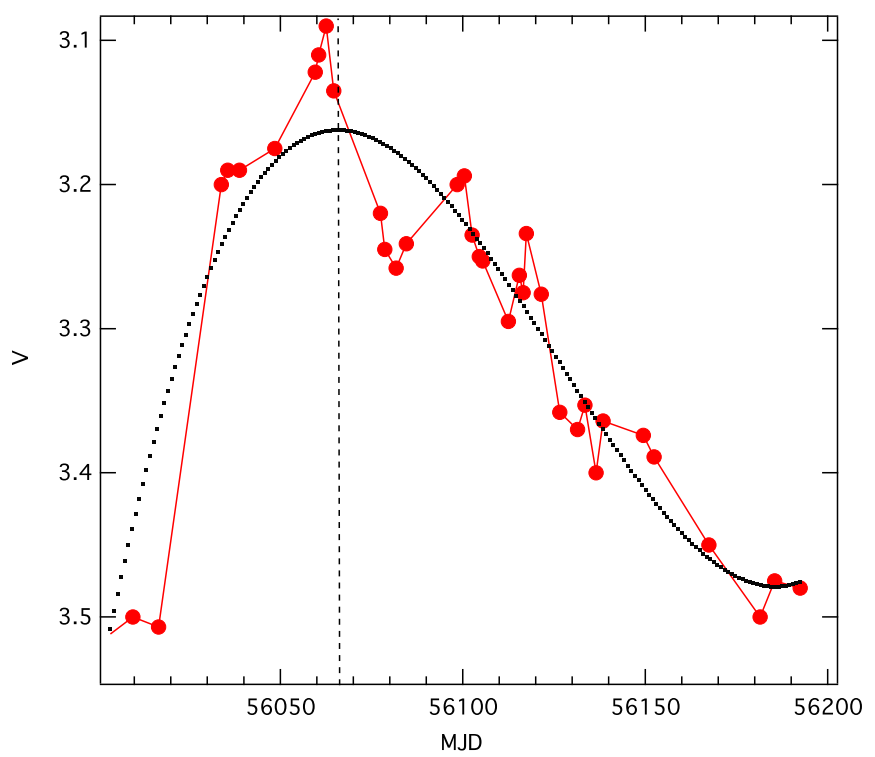

Figure 1. Outburst of $\mu$ Centauri with a peak at MJD $=56066.6$. The red curve corresponds to Argelander photometry and the dotted curve is the fit using a fifth-degree polynomial. The black dashed vertical line represents the maximum intensity of the peak.

optical lines (specially $\mathrm{H} \alpha$ ) probe the external disk regions (e.g., Haubois et al. 2014), near-infrared hydrogen lines are much better tracers for the inner parts of the disk.

However, only in recent years has the near-IR region of the spectrum been studied in greater detail. The Apache Point Observatory Galactic Evolution Experiment (APOGEE) allowed for the identification of a large number of new Be stars among objects originally targeted as telluric standard stars, with high-resolution $(\mathrm{R}=22,500) H$-band spectroscopy (Chojnowski et al. 2015). The variability of these Be stars was studied in a follow-up article (Chojnowski et al. 2017) and by Labadie-Bartz et al. (2017).

The $L$-band of the near-IR $(2.9-4.1 \mu \mathrm{m})$ has neither been studied with the same resolution nor for the same amount of targets as the $H$-band, but it has provided an excellent tool to study the environment of Be stars. The $L$-band contains important diagnostic Hydrogen lines of Bracket series $(\operatorname{Br} \alpha, \operatorname{Br} \beta)$, Pfund series $(\operatorname{Pf} \gamma)$, and also the Humphreys series, which yield kinematical and physical information of the innermost circumstellar environment, closest to the central star (Marlborough et al. 1997; Lenorzer et al. 2002a, 2002b; Mennickent et al. 2009; Granada et al. 2010). Lenorzer et al. (2002a) presented a line ratio diagram to test the density and spatial distribution of circumstellar gas around hot stars, and as proposed by Jones et al. (2009) and Mennickent et al. (2009), this method can be applied in particular to Be stars.

Thus, studying the time evolution of near-IR $L$-band lines that form in the innermost region of the disk is of particular interest to understand the formation and dissipation processes observed in Be stars. Sabogal et al. (2017) presented new $L$-band spectra of a number of Be stars and studied the long-term variability of these objects plus others available in the literature. They found some objects exhibiting disk dissipation, but none exhibiting disk formation. To our knowledge, the process of disk formation has never been observed yet in the $L$-band.

With the aim of observing a star in the disk formation process in the $L$-band, we pursued an observational campaign with the aim of tracing the formation, evolution, and dissipation of the disk of a Be star through near-IR spectroscopy. We decided to choose an early-type Be star, because observations carried out in the optical range have shown that these objects tend to present more recurrent outbursts than late-type objects and are hence more proactive to display the Be phenomenon (Rivinius et al. 2013) and references therein.

The non-radially pulsating Be star $\mu$ Centauri (B2Vnpe, $v \sin i=194 \mathrm{~km} \mathrm{~s}^{-1}$, HD $120324=$ HR 5193) was selected due to its brightness $(V \approx 3.45)$ and short recurrence time of relatively well documented outbursts (Rivinius et al. 1998a, 1998b, 2001). Particularly for $\mu$ Centauri, Hanuschik et al. (1993) studied the circumstellar emission variation in $\mathrm{H} \alpha$ region for a period of about 200 days, finding typical risings of 2-5 days. A few years later, Rivinius et al. (1998a) described the behavior of the stellar and circumstellar activity in this star, finding discrete events characterized as outbursts and concluding that $\mu$ Centauri was building a disk that was not detected between 1977 and 1989 .

Our original goal was to observe this variable object during a major outburst in order to document the evolution of the physical conditions of the envelope. Surprisingly, we found the star during a rare long quiescence stage, but more surprisingly, we detected important changes in emission line strength prior to a major outburst, which are discussed in this paper.

This paper is organized as follows: in Section 2, we present our observations, the unpublished visual observations of $\mu$ Centauri between the years 1998 and 2014, and our photometric and near-IR spectroscopic observations, methodology of spectral reduction, and analysis. In Section 3, we present the main results about line parameters and light curve analysis. We discuss and interpret these results in Section 4. Finally, we present our conclusions in Section 5 .

\section{Observations}

\subsection{Photometric Observations}

We present for the first time a total of 906 visual magnitudes of $\mu$ Centauri distributed from 1998 to 2014 . Visual photometry was performed by Sebastián Otero from Buenos Aires, Argentina using the Argelander method (Argelander 1843), which consists of the observation of a target star and non-variable stars as comparison stars with magnitudes and colors as similar as possible to the variable star. This observational method has been recently validated for bright variable stars (Štefl et al. 2003; Carciofi et al. 2012; Chesneau et al. 2014) and results compared with photoelectric photometry with a resulting accuracy of 0.05 mag or better for bright naked eye stars like $\mu$ Centauri (Otero et al. 2001; Otero \& Moon 2006); we also have compared an outburst centered at MJD $=56066.6$ (Figure 1) with the results obtained by Haubois et al. (2012).

In the case of $\mu$ Centauri, the availability of suitable comparison stars near the variable contributes to the reliability of the estimates. However, several of the comparison stars used, including the main one, $\nu$ Cen, which is only 47 arc minutes away, are variable at the $0.01-0.03 \mathrm{mag}$. level so this will contribute some scatter to the results. The estimates at the beginning and end of each season-especially before 2004, denoted as a vertical line in Figure 2-may suffer from atmospheric extinction, but over the last several years that effect has been minimized by the use of comparison stars lying at the same altitude of the variable and by averaging estimates using different comparison stars when the conditions were not ideal. This improvement to the observation method was 

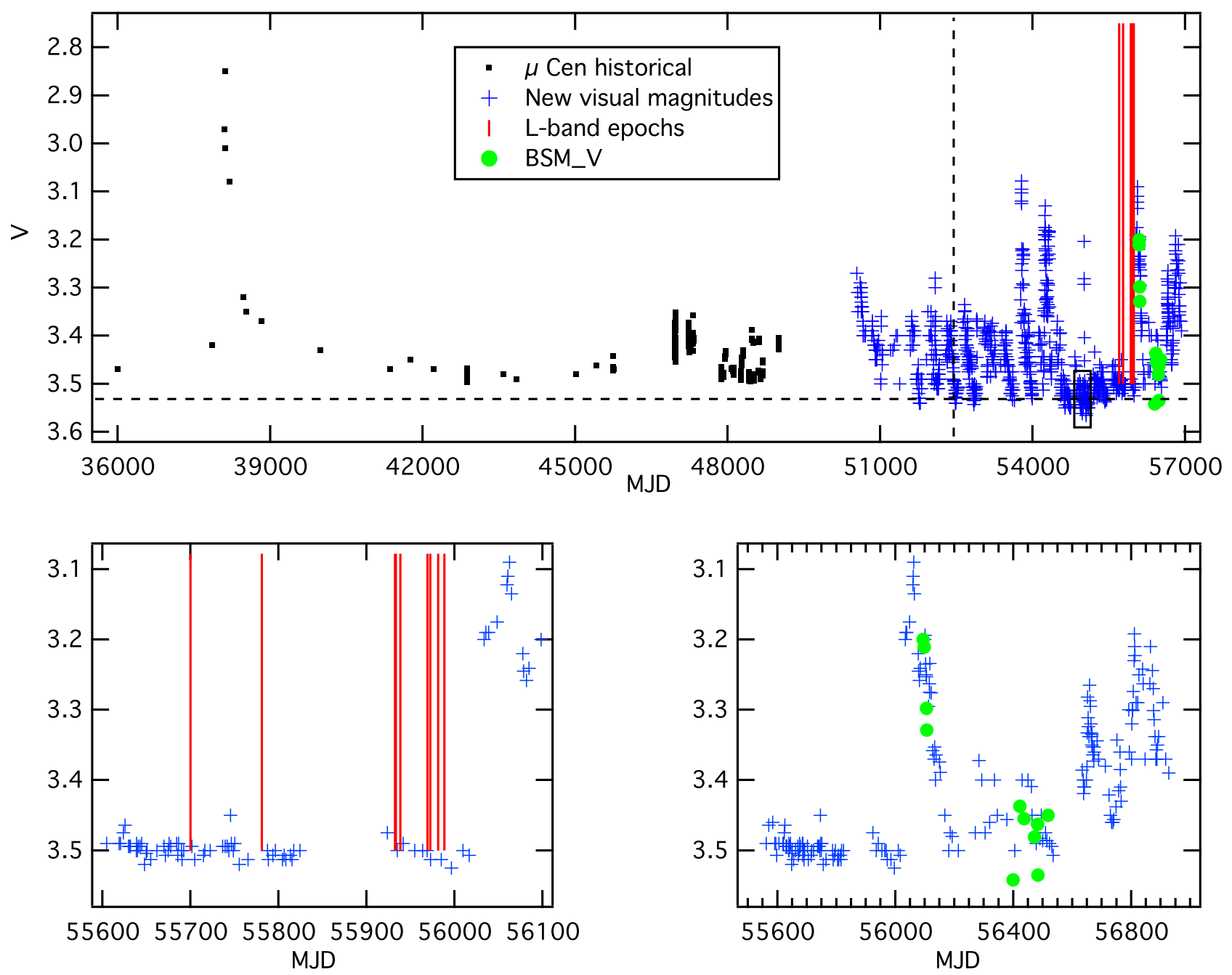

Figure 2. Top panel: light curve of $\mu$ Centauri in the $V$ band. The year 2004 is represented by the vertical line. Black dots: historical light curve compiled from different authors (Mendoza 1958; Feinstein 1968, 1975; Gutierrez-Moreno \& Moreno 1968; Percy et al. 1981; Stagg 1987; Dachs et al. 1988; Cuypers et al. 1989; Perryman et al. 1997) Blue crosses: visual observations from S. Otero using Argelander method; Red lines: epochs of our L-band spectra; Green circles: BSM photometry used for comparison with visual magnitudes. Dashed line represent historical minimum of $\mu$ Cen; this was estimated by averaging the magnitudes within the box with a resulting value of 3.53 mag. Bottom left: a zoom of historical light curve showing the correspondence between $L$-band epochs and visual photometry. Bottom right: zoom of the historical light curve showing the correspondence between BSM and Argelander photometry.

performed after 2004 and allowed us to maintain the accuracy mentioned above. Even then, only the early epochs were accurate enough to detect the presence of outbursts.

The high variability of $\mu$ Centauri due to changes in the disk and color of the star does not affect our visual measurements significantly. Comparison with simultaneous AAVSO BSM Epoch Photometry Data (A. Henden 2018, private communication-Figure 2) confirms that the accuracy is better than 0.05 mag. The comparison stars used are the same for all epochs and are listed in Table 1, and observing log is presented in Table 2. We use the correspondence between the $V$-band magnitude and our visual magnitudes based on comparison with photoelectric $\mathrm{BSO}^{6}$ photometry presented in Table 3 and previous studies indicating compatibility between the corresponding data sets as presented in Otero et al. (2001).

\subsection{Near-IR Spectroscopic Observations}

We obtained nine $L$-band spectra of $\mu$ Centauri using the Infrared Spectrometer and Array Camera (ISAAC;

\footnotetext{
6 AAVSO Bright Star Monitor-http://www.aavso.org/bsm.
}

Table 1

Comparison Stars Used for the Visual Photometric Estimates of $\mu$ Centauri Reported in This Paper

\begin{tabular}{lccc}
\hline \hline Star & $V$ & $B-V$ & Comment \\
\hline$\lambda$ Cen & 3.13 & -0.05 & no comment \\
$\kappa$ Cen & 3.14 & -0.21 & $\beta$ Cep, ampl. $0.01 \mathrm{mag}$. \\
$\nu$ Cen & 3.41 & -0.23 & $\beta$ Cep, ampl. $0.02 \mathrm{mag}$. \\
$\alpha$ Cir & 3.18 & 0.25 & roAp, ampl. 0.02 mag. \\
$\delta$ Lup & 3.22 & -0.22 & $\beta$ Cep, ampl. 0.03 mag. \\
$\epsilon$ Lup & 3.37 & -0.18 & $\beta$ Cep, ampl. 0.02 mag. \\
$\iota$ Lup & 3.55 & -0.18 & possible $\beta$ Cep, ampl. 0.01 mag. \\
$\beta$ Mus & 3.05 & -0.19 & no comment \\
\hline
\end{tabular}

Moorwood et al. 1998) located at UT-3 in Cerro Paranal ESO Observatory. Our observations span 10 months from 2011 May 18 to 2012 March 1; the used spectra is shown in Figure 3. The same configuration was used for each spectrum by applying the nodding and chopping observing mode with a central wavelength of $3.55 \mu \mathrm{m}$ and a narrow slit of 0 !" 3 providing a resolving power $R \approx 1200$. The exposure time of 
Table 2

Observing Log for $\mu$ Cen and the Telluric Standard HR 4903

\begin{tabular}{lcccc}
\hline \hline Object & MJD & Exptime $(\mathrm{s})$ & Airmass & $\mathrm{S} / \mathrm{N}$ \\
\hline$\mu$ Cen & 55699.9891 & 1.848 & 1.114 & 133.6 \\
HR 4903 & 55700.0172 & 1.848 & 1.331 & $\ldots$ \\
$\mu$ Cen & 55781.0428 & 1.848 & 1.471 & 127.4 \\
HR 4903 & 55781.0412 & 1.848 & 1.459 & $\ldots$ \\
$\mu$ Cen & 55932.3567 & 1.848 & 1.312 & 137.2 \\
HR 4903 & 55932.3434 & 1.848 & 1.216 & $\ldots$ \\
$\mu$ Cen & 55933.3129 & 1.848 & 1.594 & 95.2 \\
HR 4903 & 55933.2738 & 1.848 & 1.606 & $\ldots$ \\
$\mu$ Cen & 55938.3051 & 1.848 & 1.543 & 125.3 \\
HR 4903 & 55938.2948 & 1.848 & 1.359 & $\ldots$ \\
$\mu$ Cen & 55969.3290 & 1.848 & 1.087 & 122.5 \\
HR 4903 & 55969.3156 & 1.848 & 1.070 & $\ldots$ \\
$\mu$ Cen & 55972.3378 & 1.848 & 1.066 & 131.7 \\
HR 4903 & 55972.3216 & 1.848 & 1.063 & $\ldots$ \\
$\mu$ Cen & 55981.2482 & 1.848 & 1.205 & 137.1 \\
HR 4903 & 55981.2592 & 1.848 & 1.091 & $\ldots$ \\
$\mu$ Cen & 55988.3589 & 1.848 & 1.067 & 139.1 \\
HR 4903 & 55988.3706 & 1.848 & 1.166 & $\ldots$ \\
\hline
\end{tabular}

each spectrum was set to $1.8 \mathrm{~s}$ providing a signal-to-noise ratio $(\mathrm{S} / \mathrm{N})$ larger than 120 in regions deployed of telluric lines.

\subsection{Data Reduction}

The data were reduced using the ISAAC pipeline and Gasgano software. After reduction process, the images were telluric-corrected using the $\mathrm{IRAF}^{7}$ telluric task and the standard star HR 4903 observed at similar airmass few minutes before $\mu$ Cen. Our telluric standard is HR 4903 of spectral type G1V; this star was divided by a solar spectrum of similar spectral resolution. This reduction process is described by Maiolino et al. (1996). Flux losses due to the narrow slit and seeing variations were large and prevented us from performing a correct calibration of the stellar flux, even considering differential spectrophotometry between $\mu$ Centauri and its telluric star. The wavelength calibration allows to identify lines and determine emission line properties, but at the resolving power of $R \sim 1200$, the radial velocities are not quite accurate. For the above reasons, we decided to continuum normalize the spectra and provide information of emission line widths, equivalent widths, relative intensities, and line flux ratios. The analysis and discussion described in this paper is based on these measurements.

\section{Results \\ 3.1. Light Curve}

We present an up-to-date visual light curve of $\mu$ Centauri in Figure 2. The light curve of $\mu$ Centauri has been studied by Harmanec (1987), who found a single period of 3.535 days. A few years later, Cuypers et al. (1989) observed the star over two years (1987 and 1988) and used photometric data of 27 nights to find a double-wave light curve with periodicity of 2.1 days using Fourier periodogram analysis. Then, with data taken in 1988, it was possible to refine the period to 2.1017 days. Balona et al. (2001)

\footnotetext{
IRAF is distributed by the National Optical Astronomy Observatories, which are operated by the Association of Universities for Research in Astronomy (AURA), Inc., under cooperative agreement with the National Science Foundation.
}

also studied $\mu$ Centauri photometrically and concluded a periodicity of the order of 1 day; the result of a single period was attributed to co-rotating clouds around the central star. On the contrary, Rivinius et al. (1998a) reported the presence of six periods in $\mu$ Cen. Until now, it is clear that there has not been a well established periodicity for this star. Our visual observations are not accurate enough to perform the analysis of small amplitude non-radial pulsation frequencies. However, the visual inspection of our light curve reveals the presence of several outbursts during the last 16 years, which are well above any reasonable estimate for a single point photometric error (Figure 2). In addition to the very large outburst around $\mathrm{MJD}=37500$, we have found four major outbursts occurring around MJD 53782, 54243, 55014, and 56062; the time lapses between consecutive outbursts are 461, 771, and 1048 days, incompatible with a single recurrence time. The outburst are characterized by a sudden rise of brightness of amplitudes between 0.1 and 0.4 mag, occurring in timescales of a few days followed by a slow decline lasting $\sim 100$ days. No periodic pattern is observed in the long-term light curve. Our spectroscopic observations, obtained between JD 2455700 and 2455988, occur during a relatively long phase of quiescence, when the photometric activity, if present, is at the level of $\Delta V \approx 0.02$ mag. Just after the infrared observations, a major outburst started around JD 2456030 lasting around 150 days.

We have analyzed an outburst in order to compare the conclusions obtained by Haubois et al. (2012) with our photometric data. We have chosen an outburst centered in $\mathrm{MJD}=56066.22$ due to its strength and amplitude of $\Delta V=0.4 \mathrm{mag}$. The outburst was fitted by a fifth-degree polynomial and compared with the models done by Haubois et al. (2012). If we split the outburst at its maximum brightness, which should be where the central star stop feeding the disk, there is a build-up stage and a dissipation stage. We have found the dissipation timescale to be larger than the build-up stage, finding a build-up timescale of 46.4 days and a decay lasting 116.78 days (Figure 1). Their results are in agreement with the predictions by Haubois et al. (2012).

\subsection{Line Identification and Line Parameters}

We identify emission Hydrogen recombination lines, as the Humphreys lines from transition $14-6$ to $30-6$ (Table 4). At some epochs, we observe higher-order Humphreys lines. This is due to different conditions of the envelope and can be seen in Lenorzer diagram (5). The higher order observed was $\mathrm{Hu}(30-6)$ at dates MJD 55981.2482 and MJD 55988.3589. Also, we identify emission in $\operatorname{Br} \alpha, \operatorname{Pf} \gamma, \operatorname{Pf} \epsilon$, and $\operatorname{Pf} \delta$, displayed in Figure 3 as follows: $\operatorname{Br} \alpha$ by a black triangle, $\operatorname{Pf} \gamma$ by a gray triangle, and the Humphreys series by white triangles from the transition 14-6 beside $\operatorname{Br} \alpha$ to the end of the series. Another identified line in the first spectrum is the line He I 4.038-4.041 $\mu \mathrm{m}$ that disappeared toward the end of the observations. This line was also observed in $\mu$ Centauri and other Be stars and related objects-OZ Nor, V4024 Sgr, and V1150 Tau—by Mennickent et al. (2009)- - here and after M09- but in those observations, the emission in $\mathrm{Pf} \gamma$ and $\operatorname{Pf} \epsilon$ was not detected.

The spectra shortward of $3.5 \mu \mathrm{m}$ were not successfully corrected by telluric lines due to high variability of the atmospheric features in this region. Since diagnostic lines used for the analysis are outside this zone, we omitted the problematic region from the analysis. The target lines in this study are the Hydrogen Humphreys series, $\operatorname{Br} \alpha$ and $\operatorname{Pf} \gamma$. The measurement of the main parameters as equivalent width (EW) and full width at 


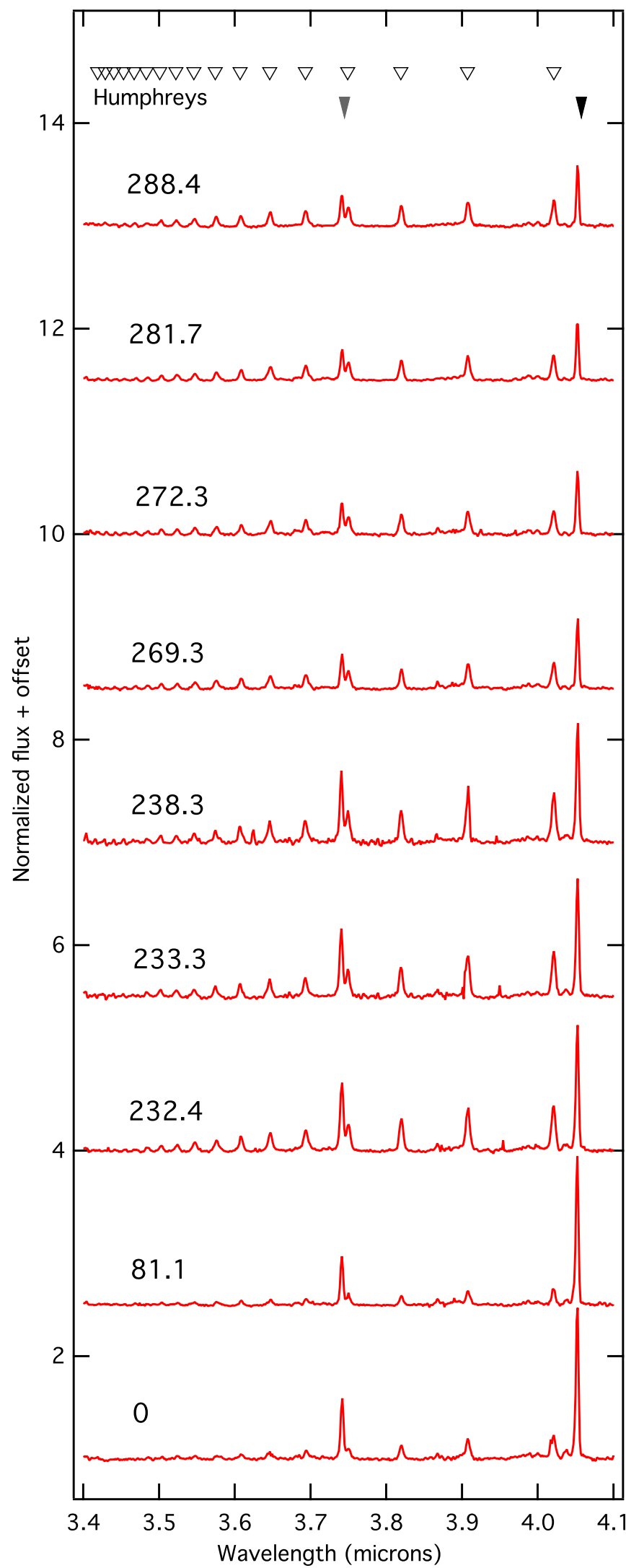

Figure 3. $L$-band spectra of $\mu$ Cen, time direction goes upward. The black arrow indicates the position of $\operatorname{Br} \alpha$ and gray arrow of $\operatorname{Pf} \gamma$. The number beside each spectra indicates the number of days from the first spectrum.
Table 3

BSM Photometry for $\mu$ Centauri Used for Comparison with Visual Magnitudes

\begin{tabular}{lccc}
\hline \hline MJD & $V$ & Verr & $B-V$ \\
\hline 56094.1326 & 3.200 & 0.007 & -0.125 \\
56098.1210 & 3.211 & 0.006 & -0.147 \\
56105.1020 & 3.298 & 0.003 & -0.168 \\
56106.1003 & 3.329 & 0.006 & -0.153 \\
56399.2881 & 3.542 & 0.002 & -0.233 \\
56422.2303 & 3.437 & 0.005 & -0.234 \\
56436.1932 & 3.455 & 0.013 & -0.223 \\
56472.1009 & 3.481 & 0.032 & -0.226 \\
56482.0747 & 3.463 & 0.005 & -0.262 \\
56483.0723 & 3.535 & 0.007 & -0.144 \\
56517.9839 & 3.450 & 0.018 & -0.144 \\
\hline
\end{tabular}

Note. A. Henden (2018, private communication).

Table 4

H I Lines Detected from 3.5 to $4.1 \mu \mathrm{m}$ in All Observations

\begin{tabular}{lccc}
\hline \hline$\lambda(\mu)$ & Transition & $\lambda(\mu)$ & Transition \\
\hline 4.052 & H I $(5-4)$ & 3.546 & H I $(22-6)$ \\
4.021 & H I $(14-6)$ & 3.522 & H I $(23-6)$ \\
3.907 & H I $(15-6)$ & 3.501 & H I $(24-6)$ \\
3.819 & H I $(16-6)$ & 3.483 & H I $(25-6)$ \\
3.749 & H I $(17-6)$ & 3.467 & H I $(26-6)$ \\
3.740 & H I $(8-5)$ & 3.453 & H I $(27-6)$ \\
3.693 & H I $(18-6)$ & 3.440 & H I $(28-6)$ \\
3.646 & H I $(19-6)$ & 3.429 & H I $(29-6)$ \\
3.607 & H I $(20-6)$ & 3.419 & H I $(30-6)$ \\
3.574 & H I $(21-6)$ & $\cdots$ & $\ldots$ \\
\hline
\end{tabular}

half maximum (FWHM) were performed using IRAF task splot, and they were measured by fitting a Gaussian to the line profile. The list of the observed lines is presented in Table 4. The values of column densities and relative extension of line forming regions were computed for different lines and epochs and tabulated in Table 5, and nightly parameter reports are given in Tables 6 and 7. The formal error of the fit for the Bracket and Pfund series is $2 \%$ in EW and $2.5 \%$ in FWHM measurement. For Humphreys, as the line fluxes are lower, the error in the measurement is higher, and we have calculated $6 \%$ for $\mathrm{EW}$ and $3.5 \%$ for FWHM of the emission lines. These errors were estimated by measuring several times the data in order to determine the standard deviation. We are aware that using this technique may underestimate the error. The measured FHWM are related to the true FWHM through the relation

$$
\mathrm{FWHM}_{\mathrm{TRUE}}=\sqrt{\mathrm{FWHM}_{\mathrm{OBS}}^{2}-\mathrm{FWHM}_{\mathrm{INS}}^{2}}
$$

where FWHM INS $_{\text {in }}$ the instrumental FWHM equal to $31 \AA$.

\subsubsection{Equivalent Widths and FWHM Behavior}

We present, for the fist time in Figure 4, the evolution for a single star between $\mathrm{EW} / \lambda$ and wavelength for the Humphreys series. There is not a well defined pattern for $\mathrm{EW} / \lambda$ along our time coverage for Humphreys lines; however, there seems to be a trend in the behavior of the Brackett $\alpha$ and Pfund $\gamma$ lines. In the first observation, there is a large difference between $\operatorname{Br} \alpha$, $\operatorname{Pf} \gamma$, and Humphreys lines, but when time goes by, EW $/ \lambda$ of 
Table 5

Hydrogen Column Densities for Level 6 and Relative Extension of the Forming Regions of Humphreys Lines

\begin{tabular}{|c|c|c|c|c|c|c|}
\hline \multirow{2}{*}{$\begin{array}{r}\lambda \\
\mu \mathrm{m}\end{array}$} & \multicolumn{2}{|c|}{55699.9891} & \multicolumn{2}{|c|}{55781.0428} & \multicolumn{2}{|c|}{55932.3567} \\
\hline & $\begin{array}{c}N_{6} \\
10^{14} \mathrm{~cm}^{-2}\end{array}$ & $\Delta R_{n} / \Delta R_{16}$ & $\begin{array}{c}N_{6} \\
10^{14} \mathrm{~cm}^{-2}\end{array}$ & $\Delta R_{n} / \Delta R_{16}$ & $\begin{array}{c}N_{6} \\
10^{14} \mathrm{~cm}^{-2}\end{array}$ & $\Delta R_{n} / \Delta R_{16}$ \\
\hline 4.021 & 0.68 & 0.37 & 1.98 & 0.59 & 1.93 & 1.55 \\
\hline 3.907 & 0.53 & 0.28 & 1.52 & 0.46 & 0.50 & 0.40 \\
\hline 3.819 & 1.83 & 1.00 & 3.33 & 1.00 & 1.25 & 1.00 \\
\hline 3.749 & 2.38 & 1.30 & 2.99 & 0.90 & 1.71 & 1.37 \\
\hline 3.693 & 3.54 & 1.93 & 1.77 & 0.53 & 1.47 & 1.18 \\
\hline$\lambda$ & \multicolumn{2}{|c|}{55933.3129} & \multicolumn{2}{|c|}{55938.3051} & \multicolumn{2}{|c|}{55969.3290} \\
\hline$\mu \mathrm{m}$ & $\begin{array}{c}N_{6} \\
10^{14} \mathrm{~cm}^{-2}\end{array}$ & $\Delta R_{n} / \Delta R_{16}$ & $\begin{array}{c}N_{6} \\
10^{14} \mathrm{~cm}^{-2}\end{array}$ & $\Delta R_{n} / \Delta R_{16}$ & $\begin{array}{c}N_{6} \\
10^{14} \mathrm{~cm}^{-2}\end{array}$ & $\Delta R_{n} / \Delta R_{16}$ \\
\hline 4.021 & 1.34 & 0.86 & 1.21 & 0.95 & 1.35 & 0.82 \\
\hline 3.907 & 0.35 & 0.22 & 0.40 & 0.31 & 1.94 & 1.18 \\
\hline 3.819 & 1.56 & 1.00 & 1.28 & 1.00 & 1.65 & 1.00 \\
\hline 3.749 & 1.13 & 0.72 & 0.15 & 0.12 & 1.10 & 0.67 \\
\hline 3.693 & 1.95 & 1.25 & 1.68 & 1.32 & $\ldots$ & $\ldots$ \\
\hline$\lambda$ & \multicolumn{2}{|c|}{55972.3378} & \multicolumn{2}{|c|}{55981.2482} & \multicolumn{2}{|c|}{55988.3589} \\
\hline$\mu \mathrm{m}$ & $\begin{array}{c}N_{6} \\
10^{14} \mathrm{~cm}^{-2}\end{array}$ & $\Delta R_{n} / \Delta R_{16}$ & $\begin{array}{c}N_{6} \\
10^{14} \mathrm{~cm}^{-2}\end{array}$ & $\Delta R_{n} / \Delta R_{16}$ & $\begin{array}{c}N_{6} \\
10^{14} \mathrm{~cm}^{-2}\end{array}$ & $\Delta R_{n} / \Delta R_{16}$ \\
\hline 4.021 & 3.01 & 1.08 & 3.05 & 0.91 & 2.36 & 0.95 \\
\hline 3.907 & 2.45 & 0.88 & 2.51 & 0.75 & 2.50 & 1.01 \\
\hline 3.819 & 2.80 & 1.00 & 3.36 & 1.00 & 2.49 & 1.00 \\
\hline 3.749 & 3.18 & 1.14 & 3.05 & 0.91 & 2.65 & 1.06 \\
\hline 3.693 & 2.04 & 0.73 & 2.16 & 0.64 & 1.65 & 0.66 \\
\hline
\end{tabular}

Table 6

Equivalent Widths in $\AA$, for Hydrogen Recombination Lines Observed

\begin{tabular}{|c|c|c|c|c|c|c|c|c|c|c|}
\hline MJD & $\mathrm{EW}_{\mathrm{Br}_{\alpha}}$ & $\mathrm{EW}_{\mathrm{Pf}_{\gamma}}$ & $\mathrm{EW}_{\mathrm{Hu}_{14}}$ & $\mathrm{EW}_{\mathrm{Hu}_{15}}$ & $\mathrm{EW}_{\mathrm{Hu}_{16}}$ & $\mathrm{EW}_{\mathrm{Hu}_{17}}$ & $\mathrm{EW}_{\mathrm{Hu}_{18}}$ & $\mathrm{EW}_{\mathrm{Hu} 19}$ & $\mathrm{EW}_{\mathrm{Hu}_{20}}$ & $\mathrm{EW}_{\mathrm{Hu}_{21}}$ \\
\hline 55699.9891 & 61.1 & 26.1 & 16.4 & 13.4 & 8.7 & 6.7 & 5.1 & 5.8 & 4.2 & 3.7 \\
\hline 55781.0428 & 64.5 & 23.3 & 10.2 & 9.6 & 6.0 & 5.4 & 5.2 & 4.9 & 4.0 & 3.7 \\
\hline 55932.3567 & 55.4 & 34.2 & 25.7 & 30.0 & 21.2 & 16.2 & 13.7 & 12.7 & 9.8 & 8.7 \\
\hline 55938.3051 & 55.2 & 33.0 & 29.7 & 28.9 & 19.7 & 18.3 & 12.7 & 11.9 & 8.9 & 8.8 \\
\hline 55969.3290 & 29.4 & 15.9 & 17.0 & 12.2 & 10.8 & 9.4 & 9.3 & 6.7 & 6.5 & 5.6 \\
\hline 55972.3378 & 26.8 & 15.1 & 15.7 & 16.2 & 13.4 & 10.8 & 10.4 & 9.9 & 6.4 & 6.3 \\
\hline MJD & $\mathrm{EW}_{\mathrm{Hu}_{22}}$ & $\mathrm{EW}_{\mathrm{Hu}_{23}}$ & $\mathrm{EW}_{\mathrm{Hu}_{24}}$ & $\mathrm{EW}_{\mathrm{Hu}_{25}}$ & $\mathrm{EW}_{\mathrm{Hu}_{26}}$ & $\mathrm{EW}_{\mathrm{Hu}_{27}}$ & $\mathrm{EW}_{\mathrm{Hu}_{28}}$ & $\mathrm{EW}_{\mathrm{Hu}_{29}}$ & $\mathrm{EW}_{\mathrm{Hu}_{30}}$ & \\
\hline 55699.9891 & 2.41 & 2.50 & 1.69 & 1.20 & $\ldots$ & $\ldots$ & $\ldots$ & $\ldots$ & $\ldots$ & \\
\hline 55781.0428 & 3.41 & 2.04 & 1.36 & $\ldots$ & $\ldots$ & $\ldots$ & $\ldots$ & $\ldots$ & $\ldots$ & \\
\hline 55932.3567 & 7.31 & 5.10 & 3.75 & 2.66 & 3.51 & 2.53 & 1.25 & $\ldots$ & $\ldots$ & \\
\hline 55933.3129 & 7.96 & 5.01 & 4.27 & 2.35 & $\ldots$ & $\ldots$ & $\ldots$ & $\ldots$ & $\ldots$ & \\
\hline 55988.3589 & 6.65 & 5.70 & 4.19 & 3.44 & $\cdots$ & $\ldots$ & $\cdots$ & $\cdots$ & $\cdots$ & \\
\hline
\end{tabular}

Note. Typical error is $6 \%$.

$\operatorname{Br} \alpha$ and $\operatorname{Pf} \gamma$ decrease, eventually reaching values close to those measured for the Humphreys lines. In addition, we observe a break up in the EW values from MJD 55938.3051 to MJD 55969.3290; these are our fifth and sixth observations. Between these two dates, the equivalent width values decrease abruptly. This is observed for all of the lines identified. The analysis of FWHM does not reveal any particular behavior along time.

\section{Discussion}

\subsection{On Emission Line Decrements}

We have measured line strengths in order to apply a diagnostic tool presented by Lenorzer et al. (2002b)-here and after $\mathrm{L02} \mathrm{a}$-where a diagram of $\log \left(\mathrm{Hu}_{14} / \mathrm{Br}_{\alpha}\right)$ versus $\log \left(\mathrm{Hu}_{14} / \mathrm{Pf}_{\gamma}\right)$ can be used to test the optical depth conditions 
Table 7

Observed FWHM in $\AA$, for Each Hydrogen Recombination Line

\begin{tabular}{|c|c|c|c|c|c|c|c|c|c|c|}
\hline MJD & $\mathrm{FWHM}_{\mathrm{Br}_{\alpha}}$ & $\mathrm{FWHM}_{\mathrm{Pf}_{\gamma}}$ & $\mathrm{FWHM}_{\mathrm{Hu}_{14}}$ & $\mathrm{FWHM}_{\mathrm{Hu}_{15}}$ & FWHM $_{\mathrm{Hu}_{16}}$ & $\mathrm{FWHM}_{\mathrm{Hu}_{17}}$ & $\mathrm{FWHM}_{\mathrm{Hu}_{18}}$ & $\mathrm{FWHM}_{\mathrm{Hu}_{19}}$ & $\mathrm{FWHM}_{\mathrm{Hu}_{20}}$ & $\mathrm{FWHM}_{\mathrm{Hu}_{21}}$ \\
\hline 55699.9891 & 38.4 & 42.0 & 76.8 & 71.9 & 60.8 & 68.4 & 60.5 & 95.5 & 92.5 & 106.3 \\
\hline 55781.0428 & 41.0 & 46.1 & 74.3 & 70.2 & 62.3 & 56.5 & 53.2 & 74.4 & 79.1 & 71.0 \\
\hline 55932.3567 & 42.0 & 49.1 & 57.2 & 69.2 & 61.4 & 59.6 & 73.1 & 71.1 & 64.9 & 81.9 \\
\hline 55933.3129 & 44.3 & 46.6 & 60.6 & 66.0 & 59.3 & 58.2 & 62.4 & 69.1 & 64.1 & 76.1 \\
\hline 55938.3051 & 44.7 & 46.2 & 63.2 & 66.7 & 62.6 & 61.4 & 68.8 & 68.0 & 62.7 & 80.8 \\
\hline 55969.3290 & 39.0 & 44.8 & 68.8 & 59.6 & 60.8 & 70.6 & 73.9 & 62.5 & 76.9 & 80.9 \\
\hline 55972.3378 & 39.6 & 45.8 & 65.7 & 70.3 & 64.9 & 62.3 & 78.6 & 75.1 & 65.8 & 74.7 \\
\hline 55981.2482 & 37.9 & 46.2 & 61.8 & 69.5 & 60.2 & 62.0 & 76.5 & 78.9 & 61.2 & 69.3 \\
\hline 55988.3589 & 37.3 & 49.0 & 62.5 & 62.0 & 58.6 & 55.7 & 65.42 & 64.7 & 61.4 & 64.6 \\
\hline MJD & $\mathrm{FWHM}_{\mathrm{Hu}_{22}}$ & $\mathrm{FWHM}_{\mathrm{Hu}_{23}}$ & $\mathrm{FWHM}_{\mathrm{Hu}_{24}}$ & $\mathrm{FWHM}_{\mathrm{Hu}_{25}}$ & $\mathrm{FWHM}_{\mathrm{Hu}_{26}}$ & $\mathrm{FWHM}_{\mathrm{Hu}_{27}}$ & $\mathrm{FWHM}_{\mathrm{Hu}_{28}}$ & $\mathrm{FWHM}_{\mathrm{Hu}_{29}}$ & $\mathrm{FWHM}_{\mathrm{Hu}_{30}}$ & \\
\hline 55699.9891 & 64.5 & 66.3 & 53.3 & 48.7 & $\cdots$ & $\ldots$ & $\cdots$ & $\ldots$ & $\ldots$ & \\
\hline 55781.0428 & 95.6 & 65.4 & 52.1 & $\ldots$ & $\ldots$ & $\ldots$ & $\ldots$ & $\ldots$ & $\ldots$ & \\
\hline 55932.3567 & 79.0 & 73.0 & 61.3 & 67.2 & 75.6 & 92.5 & 61.9 & $\ldots$ & $\ldots$ & \\
\hline 55933.3129 & 100.1 & 72.2 & 62.3 & 54.1 & $\ldots$ & $\ldots$ & $\ldots$ & $\ldots$ & $\cdots$ & \\
\hline 55938.3051 & 91.6 & 78.7 & 59.9 & 54.2 & $\ldots$ & $\ldots$ & $\cdots$ & $\ldots$ & $\ldots$ & \\
\hline 55969.3290 & 79.7 & 75.0 & 54.0 & 60.7 & 57.5 & 67.3 & $\ldots$ & $\ldots$ & $\ldots$ & \\
\hline 55972.3378 & 72.7 & 60.9 & 56.7 & 67.1 & 54.0 & 73.7 & 45.6 & 32.4 & 44.4 & \\
\hline 55981.2482 & 76.3 & 69.8 & 57.2 & 63.0 & 71.8 & 68.5 & 98.5 & 41.6 & 66.7 & \\
\hline 55988.3589 & 74.9 & 68.6 & 59.1 & $\ldots$ & $\ldots$ & $\ldots$ & $\ldots$ & $\ldots$ & $\ldots$ & \\
\hline
\end{tabular}

Note. Typical error is $3.5 \%$. 


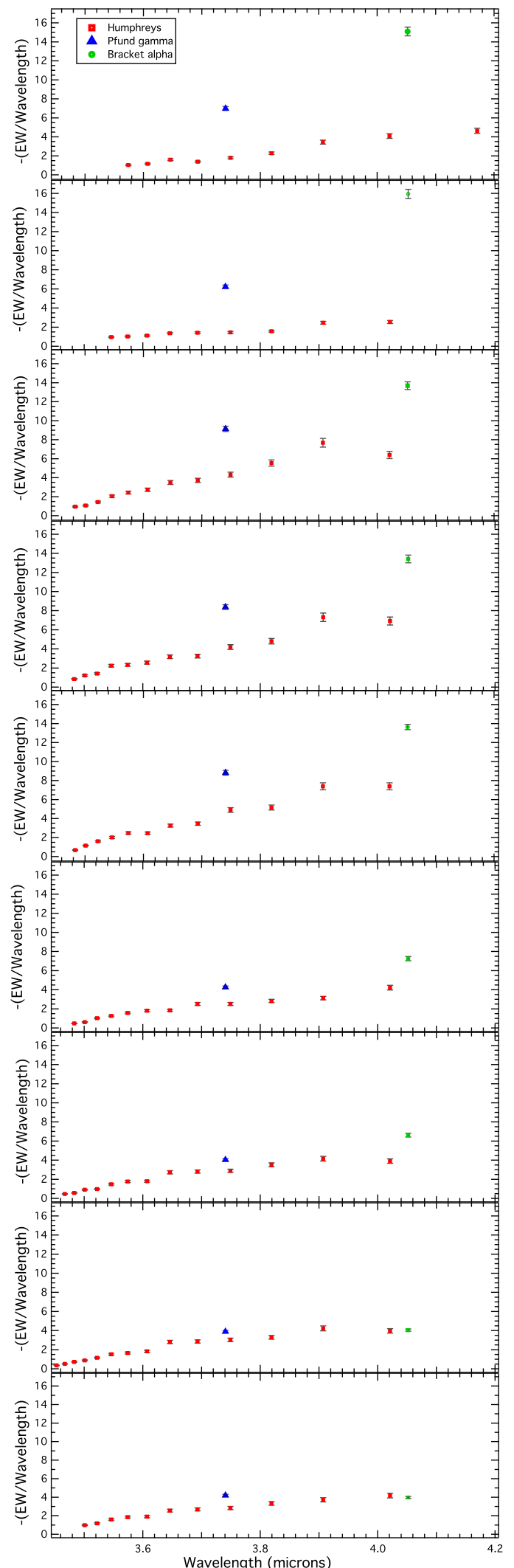

Figure 4. EW $/ \lambda$ vs. wavelength for $\operatorname{Br} \alpha, \operatorname{Pf} \gamma$, and Humphrey lines. The first observation corresponds to the lower frame and time direction goes upwards.

in a Be star disk (see also M09 and Sabogal et al. 2017). Our measurements illustrated in Figure 5 show that the position of $\mu$ Centauri varies along the diagonal of the graph by moving

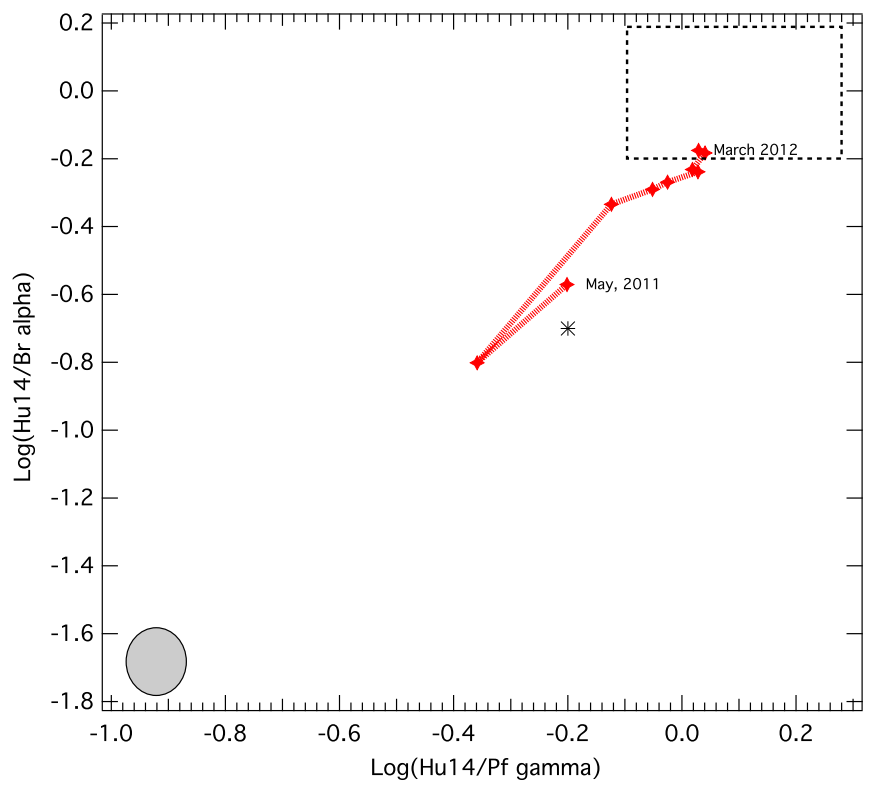

Figure 5. Line flux ratio diagram for $\log \left(\mathrm{Hu}_{14} / \mathrm{Br}_{\alpha}\right)$ vs. $\log \left(\mathrm{Hu}_{14} / \mathrm{Pf}_{\gamma}\right)$, showing the evolution track of $\mu$ Centauri during our observations. The black asterisk shows the position for $\mu$ Centauri reported by M09, the gray circle denotes the position for an optically thin case, and the dashed box represents the zone for an optically thick condition as reported by L02a.

from an optically thin zone to an optically thick one. Accordingly, the position of $\mu$ Centauri during the observation of M09 is near the optically thin region.

The correlation between EW $/ \lambda$ and wavelength in Figure 4 was reported by M09 in 13 Be stars and is also observed in $\gamma$ Cas (Hony et al. 2000). The change in emission intensities between $\mathrm{Pf}_{\gamma}$ and $\mathrm{Br}_{\alpha}$ lines relative to those of Humphreys lines is consistent with a transition from group II to group I according to M09 classification, i.e., a transition from an optically thin to optically thick circumstellar envelope.

Observing the light curve of $\mu$ Centauri in Figure 2 (top panel), we do not see large variability of the $V$ magnitude during the time span of the observations (the list of visual magnitudes is displayed in Table 8 within the Appendix). However, the $L$-band spectra display a huge variability in the intensity of Humphreys series, $\operatorname{Br} \alpha$, and $\operatorname{Pf} \gamma$ lines. This behavior is reflected in Figures 4 and 5, where the line changes are displayed.

\subsection{Estimation of Column Densities and Extension of Line- forming Regions}

Following the approximative but insightful procedure presented in Granada et al. (2010), we have estimated atomic column densities and the relative extensions for the forming region of Humphreys lines. For those Humphreys' lines with fluxes that deviate from the ones corresponding to the optically thin case, rough estimates of the atomic column density and the extension of the line-forming regions can be made. Figure 6 shows the line flux ratios for different Humphreys' lines for different epochs together with the theoretical optical thin case (Baker \& Menzel 1938).

We see that at all dates, Humphreys lines present a certain degree of departure from the optically thin case. This is also indicated in Figure 6 by the line flux ratio of $\mathrm{Hu}_{n} / \mathrm{Hu}_{16}$, which is a good indicator of the envelope optical depth (Hamann \& Simon 1987; 


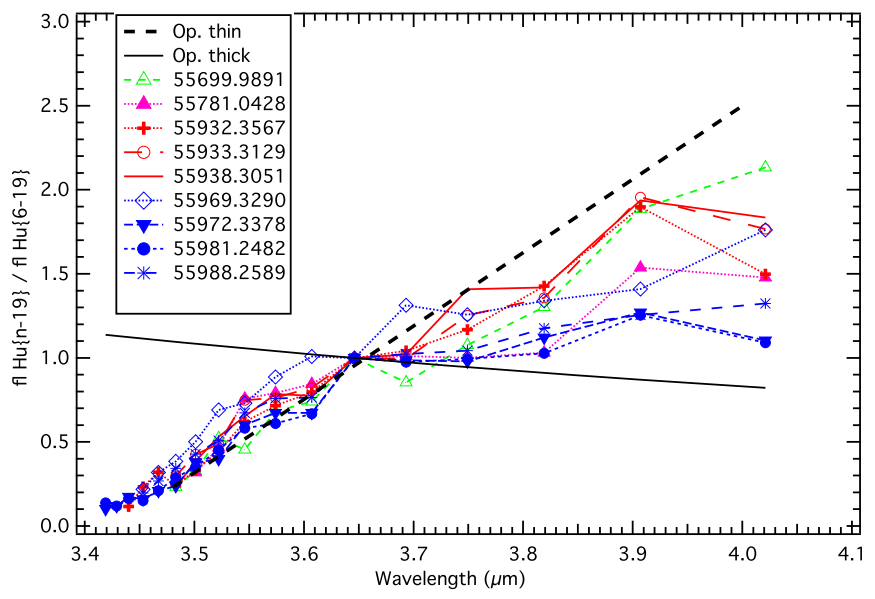

Figure 6. Line flux ratios of Humphreys' lines relative to $\mathrm{Hu}_{19}$. Different symbols indicate different epochs. The optically thin case is represented with a dashed straight line, and the optically thick case with a solid line.

Hummer \& Storey 1987), and theoretically it is found to be close to unity for optically thick envelopes. Thus, we use the following expression to estimate atomic column densities of the lower level of the transition, from different lines,

$$
N_{i}=\ln \left(\frac{\mathrm{Fl}_{\mathrm{Hu}_{n \text { thin }}}}{\mathrm{Fl}_{\mathrm{Hu}_{n}}}\right) \frac{m_{e} c \Delta \nu_{D}}{\sqrt{\pi} e^{2} f_{i}},
$$

where $m_{e}$ and $e$ are the electron mass and charge, respectively, $c$ is the speed of light, $f_{i}$ is the line oscillator strength, $\mathrm{Fl}_{\mathrm{Hu}_{n}}$ is the measured flux of the nth Humphreys line, and $\Delta \nu_{D}$ is the Doppler width of the line in frequency units. Considering that rotation is the main broadening mechanism, the broadening in velocity is taken as $V \sin i=194 \mathrm{~km} \mathrm{~s}^{-1}$ (Bernacca \& Perinotto 1970).

Since Humphreys lines are formed in the inner regions of the disk and several studies show that volumetric density varies with a lower exponent within three stellar radii (e.g., Zorec et al. 2007), we now consider that volumetric density is constant throughout all Humphreys' line-forming regions. By taking this into account if we observe differences in column densities, those differences are related to changes in the extension of line-forming regions. Then we can obtain the extension of line-forming regions, relative to one of them:

$$
\frac{\Delta R_{\mathrm{Hu}_{n}}}{\Delta R_{\mathrm{Hu}_{16}}}=\frac{\Delta N_{i_{\mathrm{Hu}_{n}}}}{\Delta N_{i_{\mathrm{Hu}_{16}}}} .
$$

The values of column densities and relative extension of line-forming regions are plotted in Figure 7. We estimate the uncertainty in the determination of $N_{i}$ is $40 \%$, and $20 \%$ for relative extension.

We find that the column densities vary in time within different epochs. In the first observation, the large column density differences with wavelength suggest that the assumption of constant density could be inadequate, because a density gradient in the Humphreys forming region could exist. The fact that the FWHM of Humphreys lines has a wavelength dependency at this epoch supports the conjecture that the line forms in somehow different conditions. In other

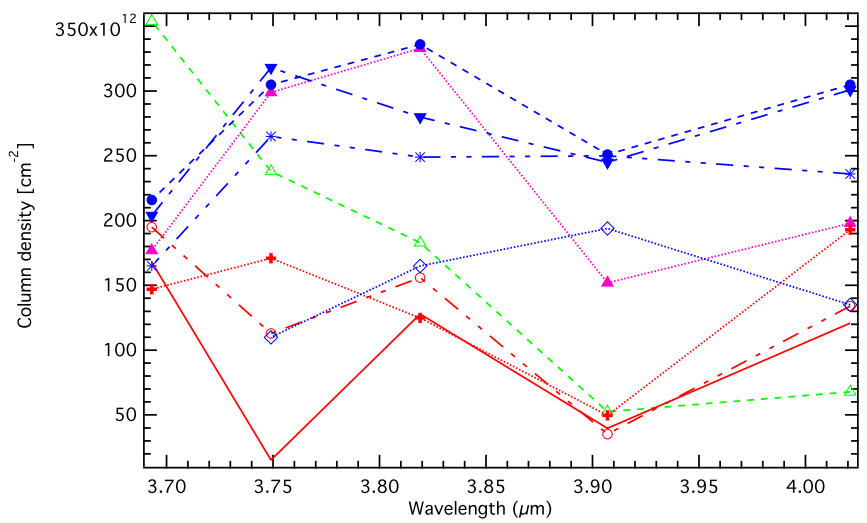

Figure 7. Atomic column densities (left), obtained from different lines and epochs: green corresponds to 2011 May 18, pink to 2011 August 08, red from 2012 January 06 to 2012 January 12, and blue from to 2012 February 12 to 2012 March 01.

epochs, the column densities exhibit a less dramatic behavior with wavelength (or at least they do not show a clear trend), and the FWHM remains within a smaller range for each observation.

During the last epoch, indicated by blue in our plots, an increase in the column density of $1.5 \times 10^{14} \mathrm{~cm}^{-2}$ (or an increase in a factor of $\sim 2$ ) occurred in 2 days. Because the rotational conditions of the line-forming region did not change significantly during these two days, we can assume that the variation in column density was due to a change in the (volumetric) density of the medium, or equivalently, due to a variation of the mass in the circumstellar envelope. However, we have to keep in mind that we obtain the column density of hydrogen atoms at level 6 (Humphreys series), so we cannot directly derive the total amount of mass injected. We could imagine though, that whatever the mass in the inner regions of the disk was, it was multiplied by a factor of 2 .

\subsection{Continuum Variability}

We have calculated a flux ratio $B_{L} / R_{L}$ where $B_{L}$ and $R_{L}$ are blue and red integrated fluxes in $L$-band zones depleted of emission lines. For that, we have calculated for each epoch the ratio between the spectra of $\mu$ Centauri and the standard star HR4903 and then calculated $S_{\lambda}$ as this ratio multiplied by a blackbody spectrum of the same temperature that the effective temperature that HR4903. Hence, we obtained a set of spectra whose flux zero point is not constrained but with slopes roughly preserving the characteristics of the original spectra. In these spectra, we calculated the flux ratio as

$$
\frac{B_{L}}{R_{L}}=\frac{\int_{3.40}^{3.45} S_{\lambda} \cdot d \lambda}{\int_{3.91}^{3.95} S_{\lambda} \cdot d \lambda}
$$

The evolution of $B_{L} / R_{L}$ throughout time is displayed in Figure 8 (left). For $\mu$ Centauri, this flux ratio turns to be bluer toward the end of the period observed. We observe a reddening in comparison with the expected value for a blackbody radiator with the same temperature that the effective temperature of $\mu$ Centauri, and this reddening is in agreement with a disk obscuring the central star. 

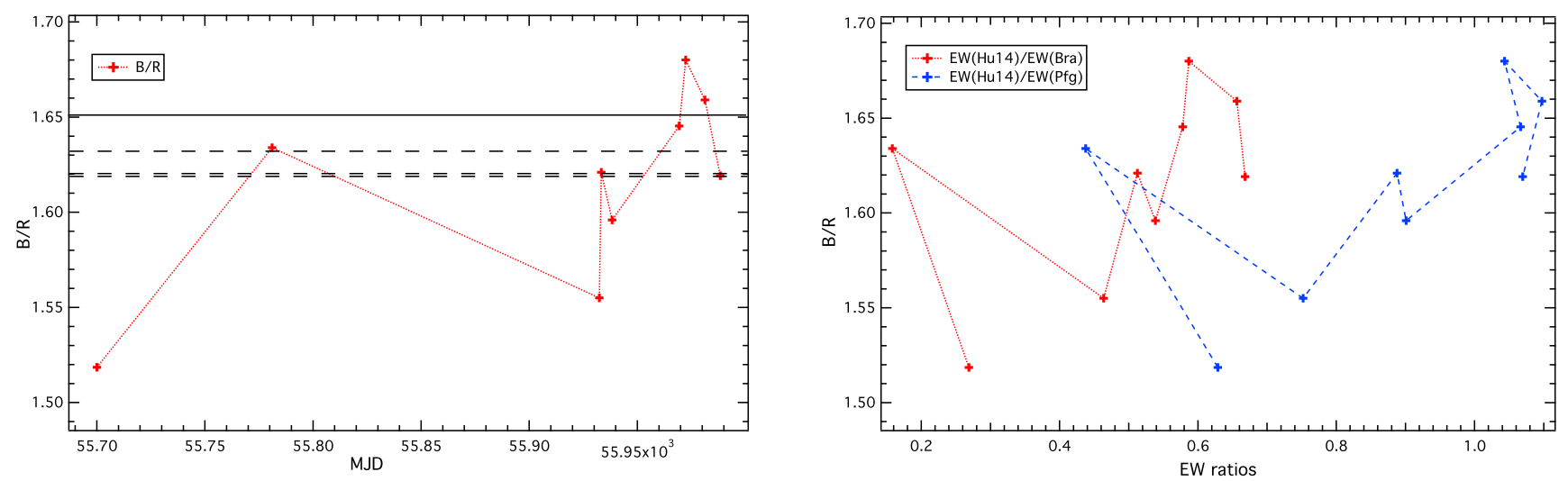

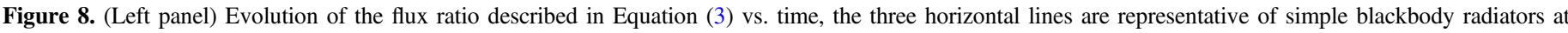

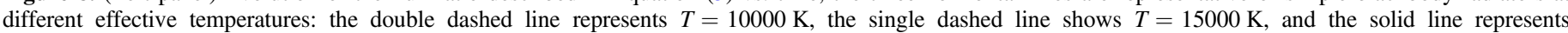

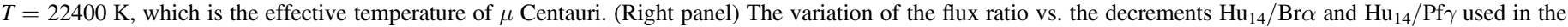
Lenorzer diagram.

The emission lines observed in the $L$-band and the variation of their strengths indicate that even at optical quiescence there is an active envelope around $\mu$ Centauri. Because of the relatively small inclination of this star (Hanuschik 1989), it is expected that the continuum intensity contribution from the central star is the same for all of the observations. Then, the observed variability between epochs is rather linked to circumstellar disk changes.

We find an overall increase in $B_{L} / R_{L}$, which means the spectra become bluer toward the end of our near-IR spectroscopic campaign. The global tendency of $B_{L} / R_{L}$ increasing before a major outburst, or equivalently the reduction of disk continuum emission, in coincidence with the presence of more compact and optically thick $L$-band hydrogen line emitting regions, could be an indication of a precursor stage, similar to the cycle of slow recovery to the quiescence magnitude prior to outburst, as described by Rivinius et al. (1998a) and as expected for a star about to start developing a CMD loop due to disk build-up (de Wit et al. 2006). Unfortunately, our time sampling is too limited to provide a precise temporal behavior of the system.

The use of the $L$-band can be an excellent tool in the study Be star disks, and we expect that fully covering an outburst episode would provide significant insight into the $\mathrm{Be}$ phenomenon.

\section{Conclusions}

We have performed for the first time a multi-epoch spectroscopic monitoring of a Be star in the $L$-band region of the near-IR, which is particularly sensitive to physical conditions of the innermost region of circumstellar envelope. We report these observations of the Be star $\mu$ Centauri together with long-term visual photometry, showing relatively frequent outburst episodes that are non-periodic and stochastic in nature, over 16 years. Even when the $V$ magnitude remained practically at a constant level or without significant changes in brightness during almost one year of our spectroscopic observations, we do observe considerable variability in the intensity of $\mathrm{H}$ I recombination lines sensitive to optical depth changes. These changes are consistent with the transition from an optically thin to an optically thick stage observed toward the end of our spectroscopic observing campaign. We also analyzed the near-IR continuum slope variability, finding that the system is bluer when the line emission is optically thick, preceding the major outburst. Because of the gap of a couple of weeks between our near-IR observations and the detection of the outburst, we cannot affirm that the observed changes in our near-IR spectra and the major outburst are causally connected. However, if they were indeed connected, our IR spectra would be the first observations of a disk building phase in the $L$-band. Moreover, because these observations trace the changes of the innermost disk regions close to the star, these changes would correspond to very early epochs in the disk build-up. Certainly, new multi-epoch and multi-wavelength monitoring of $\mu$ Centauri and other Be stars, together with detailed line modeling, are necessary to understand the disk build-up process.

This investigation was supported by CONICYT-PCHA/ Magister Nacional/2013-22131271. R.E.M. gratefully acknowledges support by VRID-Enlace 218.016.004-1.0 and the Chilean Centro de Astrofisica y Tecnologias Afines (CATA) BASAL grant AFB-170002. A.G. acknowledges the Swiss National Science Foundation, Advanced Postdoc Mobility Grant number P300P2_158443 and grant VRID 218.016.004-1.0.

\section{Appendix Photometry Tables}


Table 8

Visual Photometry for $\mu$ Centauri

\begin{tabular}{lcc}
\hline \hline MJD & $\begin{array}{c}\text { Visual } \\
\text { Magnitude } \\
\text { (mag.) }\end{array}$ & $\begin{array}{c}\text { Visual } \\
\text { Error } \\
\text { (mag.) }\end{array}$ \\
\hline 50537.7080 & 3.270 & 0.10 \\
50558.6900 & 3.310 & 0.08 \\
50564.6800 & 3.350 & 0.08 \\
50608.6041 & 3.290 & 0.08 \\
50609.6666 & 3.300 & 0.08 \\
\hline
\end{tabular}

(This table is available in its entirety in machine-readable form.)

\section{ORCID iDs}

G. Aguayo (1) https://orcid.org/0000-0003-1784-7200

\section{References}

Argelander, F. W. A. 1843, (Berlin: S. Schropp) XIX, 119 ; in 8.; DCCC.8.6, Baade, D. 1984, A\&A, 135, 101

Baker, J. G., \& Menzel, D. H. 1938, ApJ, 88, 52

Balona, L. A., James, D. J., Lawson, W. A., \& Shobbrook, R. R. 2001, MNRAS, 324, 1041

Bernacca, P. L., \& Perinotto, M. 1970, CoAsi, 239, 1

Bjorkman, J. E. 1997, in Stellar Atmospheres: Theory and Observations, ed. J. P. De Greve, R. Blomme, \& H. Hensberge (Berlin: Springer), 239

Bjorkman, J. E., \& Carciofi, A. C. 2005, in The Nature and Evolution of Disks Around Hot Stars 337, 75

Carciofi, A. C., Bjorkman, J. E., Otero, S. A., et al. 2012, ApJL, 744, L15

Chesneau, O., Meilland, A., Chapellier, E., et al. 2014, A\&A, 563, A71

Chojnowski, S. D., Whelan, D. G., Wisniewski, J. P., et al. 2015, AJ, 149, 7

Chojnowski, S. D., Wisniewski, J. P., Whelan, D. G., et al. 2017, AJ, 153, 174

Cuypers, J., Balona, L. A., \& Marang, F. 1989, A\&AS, 81, 151

Dachs, J., Kiehling, R., \& Engels, D. 1988, A\&A, 194, 167

de Wit, W. J., Lamers, H. J. G. L. M., Marquette, J. B., \& Beaulieu, J. P. 2006, A\&A, 456, 1027

Dougherty, S. M., Waters, L. B. F. M., Burki, G., et al. 1994, A\&A, 290, 609

Draper, Z. H., Wisniewski, J. P., Bjorkman, K. S., et al. 2014, ApJ, 786, 120

Feinstein, A. 1968, ZA, 68, 29

Feinstein, A. 1975, PASP, 87, 603

Frémat, Y., Zorec, J., Hubert, A.-M., \& Floquet, M. 2005, A\&A, 440, 305

Granada, A., Arias, M. L., \& Cidale, L. S. 2010, AJ, 139, 1983

Gutierrez-Moreno, A., \& Moreno, H. 1968, ApJS, 15, 459
Hamann, F., \& Simon, M. 1987, ApJ, 318, 356

Hanuschik, R. W. 1989, ApJS, 161, 61

Hanuschik, R. W., Dachs, J., Baudzus, M., \& Thimm, G. 1993, A\&A, 274, 356

Harmanec, P. 1983, HvaOB, 7, 55

Harmanec, P. 1987, IBVS, 3097, 1

Haubois, X., Carciofi, A. C., Rivinius, T., Okazaki, A. T., \& Bjorkman, J. E. 2012, ApJ, 756, 156

Haubois, X., Mota, B. C., Carciofi, A. C., et al. 2014, ApJ, 785, 12

Hony, S., Waters, L. B. F. M., Zaal, P. A., et al. 2000, A\&A, 355, 187

Hummer, D. G., \& Storey, P. J. 1987, MNRAS, 224, 801

Jaschek, M., Slettebak, A., \& Jaschek, C. 1981, BeSN, 4, 9

Jones, C. E., Molak, A., Sigut, T. A. A., et al. 2009, MNRAS, 392, 383

Labadie-Bartz, J., Chojnowski, S. D., Whelan, D. G., et al. 2017, arXiv:1708. 02594

Lee, U., Osaki, Y., \& Saio, H. 1991, MNRAS, 250, 432

Lenorzer, A., de Koter, A., \& Waters, L. B. F. M. 2002a, A\&A, 386, L5

Lenorzer, A., Vandenbussche, B., Morris, P., et al. 2002b, A\&A, 384, 473

Maiolino, R., Rieke, G. H., \& Rieke, M. J. 1996, AJ, 111, 537

Marlborough, J. M., Zijlstra, J.-W., \& Waters, L. B. F. M. 1997, A\&A, 321,867

Martayan, C., Frémat, Y., Hubert, A.-M., et al. 2007, A\&A, 462, 683

Mendoza, V. E. E. 1958, ApJ, 128, 207

Mennickent, R. E., Sabogal, B., Granada, A., \& Cidale, L. 2009, PASP, 121,125

Moorwood, A., Cuby, J.-G., Biereichel, P., et al. 1998, Msngr, 94, 7

Okazaki, A. T., Bate, M. R., Ogilvie, G. I., \& Pringle, J. E. 2002, MNRAS, 337, 967

Otero, S., Fraser, B., \& Lloyd, C. 2001, IBVS, 5026, 1

Otero, S. A., \& Moon, T. 2006, JAVSO, 34, 156

Percy, J. R., Jakate, S. M., \& Matthews, J. M. 1981, AJ, 86, 53

Perryman, M. A. C., Lindegren, L., Kovalevsky, J., et al. 1997, A\&A, 323, L49

Porter, J. M. 1999, A\&A, 348, 512

Porter, J. M., \& Rivinius, T. 2003, PASP, 115, 1153

Rímulo, L. R., Carciofi, A. C., Rivinius, T., \& Haubois, X. 2015, in IAU Symp. Vol. 307, New windows on massive stars: asteroseismology, interferometry, and spectropolarimetry (Cambridge: Cambridge Univ. Press), 133

Rivinius, T., Baade, D., Stefl, S., et al. 1998a, A\&A, 333, 125

Rivinius, T., Baade, D., Stefl, S., et al. 1998b, A\&A, 336, 177

Rivinius, T., Baade, D., Štefl, S., et al. 2001, A\&A, 369, 1058

Rivinius, T., Carciofi, A. C., \& Martayan, C. 2013, A\&ARv, 21, 69

Sabogal, B. E., Ubaque, K. Y., García-Varela, A., Álvarez, M., \& Salas, L. 2017, PASP, 129, 014203

Sigut, T. A. A., \& Patel, P. 2013, ApJ, 765, 41

Stagg, C. 1987, MNRAS, 227, 213

Stefl, S., Baade, D., Rivinius, T., et al. 2003, A\&A, 402, 253

Townsend, R. H. D., Owocki, S. P., \& Howarth, I. D. 2004, MNRAS, 350, 189

Vieira, R. G., Carciofi, A. C., Bjorkman, J. E., et al. 2017, MNRAS, 464, 3071

Zorec, J., Arias, M. L., Cidale, L., \& Ringuelet, A. E. 2007, A\&A, 470, 239 